\title{
SOME INTERSECTION THEOREMS ON TWO-VALUED FUNCTIONS
}

\author{
R. J. FAUDREE, R. H. SCHELP and VERA T. SÓS \\ Received 24 June 1983
}

Let $\mathscr{A}$ be a family of two-valued functions defined on an $n$-element set in which each pair of functions in $\mathscr{A}$ s.tisfy a given intersection condition. For certain intersection conditions we determine the miximal value of $|\mathscr{A}|$.

The study of intersection theorems for systems was started by de Bruijn and Erdös [2] and Erdös, Ko and Rado [4]. In the last twenty years a wide theory developed.

A typical general problem is the following: Let $\mathscr{L}$ be a given set of integers and $\mathscr{A} \subseteq P(S)$ satisfying

$$
\left|A_{i} \cap A_{j}\right| \in \mathscr{L} \text { for } 1 \leqq i<j \leqq m, A_{i}, A_{j} \in \mathscr{A} .
$$

How large can $m$ be under this condition?

Thus we have a condition on the size of $\left|A_{i} \cap A_{j}\right|$.

The general problem is the following: Let $S$ be an $n$-element set and $J$ be a family of subsets of $S$. The family $J$ will be called the intersection family.

\section{Strong intersection problem}

Let $\mathscr{A} \subseteq P(S)$ satisfying

$$
A_{i} \cap A_{j} \in J \text { for } 1 \leqq i<j \leqq m, A_{i}, A_{j} \in \mathscr{A} .
$$

For $n$ and $J$ fixed let $f(n ; J)$ denote the cardinality of the largest family $\mathscr{A}$ satisfying (1). Determine $f(n ; J)$.

An important subcase is the following:

\section{Weak intersection problem}

Using the same notation as above, let $\mathscr{A} \leqq P(S)$ satisfying for $A_{i}, A_{j} \in \mathscr{A}$.

$$
A_{i} \cap A_{j} \supseteqq I \text { for some } I \in J \text {. }
$$

Let $g(n ; J)$ denote the cardinality of the largest family $\mathscr{A}$ satisfying (2). Determine $g(n ; J)$. 
In some cases the family $\mathscr{A} \subseteq P(S)$ (or one of the families) of maximum cardinality is a so called kernel system which in the weak intersection case means that

$$
\bigcap_{i=1}^{m} A_{i} \in J \text { for } \quad 1 \leqq i \leqq m
$$

Obviously this property implies the intersection property (2). In the general case (strong intersection problem) (3) does not automatically imply the intersection property (1). Still, for the most part, it gives enough information to get the extremal system.

Structural type intersection theorems started in the paper of Simonovits and Sós [9] were followed by several others, e.g. [3, 5, 6, 7, 8, 10].

Tne structures considered are mostly graphs or subsets of integers and the intersection properties are given in graph theoretical or arithmetical terms.

As a simple special case of a weak intersection problem P. Erdös asked whether a family of subsets $\mathscr{A}=\left\{A_{1}, \ldots, A_{m}\right\}$ of $S=\{1, \ldots, n\}$ with the property that each pair of sets in $\mathscr{A}$ contains two consecutive integers must satisfy $m \leqq 2^{n-2}$. The following clever argument by $\mathrm{R}$. Graham verifies this fact.

Consider a family $\mathscr{A} \subseteq P(S)$ satisfying the above intersection property. Let $E$ be the set of even integers in $S$ and $O$ be the set of odd integers. For $A_{i} \in \mathscr{A}$ let $E_{i}=A_{i} \cap E$ and $O_{i}=A_{i} \cap O$. The intersection property implies that $E_{i} \cap E_{j} \neq \emptyset$ and $O_{i} \cap O_{i} \neq \emptyset$. Tuus $\left|\left\{E_{i}\right\}\right| \leqq 1 / 2\left(2^{\mid E !}\right)$ and $\left|\left\{O_{i}\right\}\right| \leqq 1 / 2\left(2^{|O|}\right)$, from which $|\mathscr{A}| \leqq(1 / 4)\left(2^{|O|+|E|}\right)=2^{1-2}$. Obviously this result is sharp, for example, the kernel system of all subsets of $S$ containing both 1 and 2 is an extremal one.

If the integers in $S$ are considered modulo $n$ (i.e. $S$ is considered as a circular sequence, 1 and $n$ are considered as consecutive elements), then the previous argument gives the same result when $n$ is even, but not when $n$ is odd.

Two natural generalizations of the above result will be considered here.

The following notation will be used throughout the paper, unless otherwise stipulated:

$X$ will denote an $n$-element set,

$X_{1}, X_{2}, \ldots, X_{l}$ will be a partition of $X$ into $l$ nonempty sets,

$k$ will be a fixed positive integer less than or equal to $l$, and $\mathscr{F}$ will be the set of all functions from $X$ into $\{0,1\}$.

In the principal results we will consider a family $\mathscr{A}$ of elements of $\mathscr{F}$ which satisfy one of the following intersection properties:

$\left(\mathrm{I}_{1}\right)$ For each $f$ and $g$ in $\mathscr{A}$, there are $k$ consecutive sets $X_{j}, X_{j+1}, \ldots, X_{j+k-1}$ (the indices of the partition $\left\{X_{1}, X_{2}, \ldots, X_{l}\right\}$ are taken modulo $l$ ) and elements $s_{i} \in X_{i}$ such that $f\left(s_{i}\right)=g\left(s_{i}\right)$ for each, $i, j \leqq i \leqq j+k-1$.

$\left(\mathrm{I}_{2}\right)$ For each $f$ and $g$ in $\mathscr{A}$ there are $k$ sets $X_{j(1)}, \ldots, X_{j(k)}$ of the partition $\left\{X_{1}, \ldots, X_{\imath}\right\}$ and elements $s_{t} \in X_{j(i)}$ such that $f\left(s_{i}\right)=g\left(s_{i}\right)$ for each $i, 1 \leqq i \leqq k$. 


\section{Results}

Theorem 1. If $\mathscr{A} \subseteq \mathscr{F}$ satisfies property $\left(\mathrm{I}_{1}\right)$, then $|\mathscr{A}| \leqq 2^{n-k}$.

Note that the bound on $|\mathscr{A}|$ in Theorem 1 is the best possible, since there is a family of $2^{n-k}$ functions which satisfy property $\left(I_{1}\right)$. Select $k$ elements $s_{1}, s_{2}, \ldots, s_{k}$ such that $s_{i} \in X_{i},(1 \leqq i \leqq k)$. The set $\mathscr{A}$ of all functions on $X$ with value 1 on $\left\{s_{i}, \ldots, s_{k}\right\}$, satisfies property $\left(\mathrm{I}_{1}\right)$ and has $2^{n-k}$ elements.

To each subset $\mathscr{A}^{*}$ of $P(X)$ we can consider the set of characteristic functions of sets in $\mathscr{A}^{*}$. Thus corresponding to $\mathscr{A}^{*}$ there is a subset $\mathscr{A}$ of $\mathscr{F}$. If each pair of elements of $\mathscr{A}^{*}$ have elements in common from $k$ consecutive sets, then $\mathscr{A}$ satisfies property $\left(\mathrm{I}_{1}\right)$. Thus the following corollary of Theorem 1 gives a generalization of the result stated in the introduction.

Corollary 2. If $\mathscr{A} \subseteq P(X)$ such that each pair of elements of $\mathscr{A}$ have elements in common from $k$ consecutive terms of $X_{1}, X_{2}, \ldots, X_{l}$ (indices taken modulo $l$ ), then $|\mathscr{A}| \leqq 2^{n-k}$.

Theorem 3. If $\mathscr{A} \subseteq \mathscr{F}$ satisfies property $\left(\mathrm{I}_{2}\right)$, then

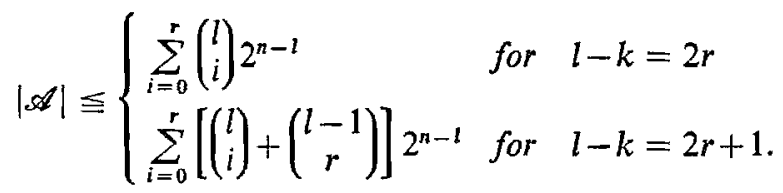

The bound on $|\mathscr{A}|$ in Theorem 3 is also sharp. To exhibit a set $\mathscr{A}$ of appropriate cardinality satisfying property $\left(\mathrm{I}_{2}\right)$, select $l$ fixed elements $Y=\left\{y_{1}, \ldots, y_{l}\right\}$ such that $y_{i} \in X_{i}$. Let $\mathscr{A}_{1}$ be the set of all functions in $\mathscr{F}$ which have value 1 on at least $l-r$ elements of $Y$, and let $\mathscr{A}_{2}$ be those functions with value 0 at $y_{1}$ and precisely $l-r-1$ values of 1 on the remaining elements of $Y$. The set $\mathscr{A}=\mathscr{A}_{1}$ when $l-k=2 r$ and $\mathscr{A}=\mathscr{A}_{1} \cup \mathscr{A}_{2}$ when $l-k=2 r+1$ has the desired properties.

Corollary 4. If $\mathscr{A} \subseteq P(X)$ such that each pair or elements of $\mathscr{A}$ have elements in common from at least $k$ terms of $X_{1}, \ldots, X_{i}$, then $|\mathscr{A}|$ satisfies (4).

Corollary 4 was also proved independently by F. R. K. Chung; R. L. Graham, P. Frankl and J. Shearer.

There is an interesting question involving a strengthening of Theorem 1 . Consider fixed positive integers $a_{1}<a_{2}<\ldots<a_{k} \leqq l$, and the following intersection property which generalizes property $\left(I_{1}\right)$.

$\left(\mathrm{I}_{3}\right)$ For each $f$ and $g$ in $\mathscr{A}$, there is a non-negative integer $t$ which determines a subsequence $X_{a_{1}+t}, X_{a_{2}+t}, \ldots, X_{a_{k}+t}$ (the indices taken modulo 1 ) of the sequence $X_{1}, X_{2}, \ldots, X_{i}$ and elements $s_{i} \in X_{a_{i}+t}$ such that $f\left(s_{i}\right)=g\left(s_{i}\right)$ for each $i$, $(1 \leqq i \leqq k)$.

Note that $\left(\mathrm{I}_{3}\right)$ is just $\left(\mathrm{I}_{1}\right)$ when $a_{i}=i$ for each $i$. It seems reasonable that the conclusion of Theorem 1 will hold when $\left(I_{1}\right)$ is replaced by $\left(I_{3}\right)$. Thus we state the following conjecture.

Conjecture. If $\mathscr{A} \subseteq \mathscr{F}$ satifies property $\left(\mathrm{I}_{3}\right)$, then $|\mathscr{A}| \leqq 2^{n-k}$. 

jecture.

The following special result also gives some evidence in support of the con-

Theorem 5. Let $a_{1}<a_{2}<a_{3}$ be elements of $X=\{1,2, \ldots, n\}$. If $\mathscr{A} \subseteq \mathscr{F}$ is such that for each pair $f, g \in \mathscr{A}, f\left(a_{i}+t\right)=g\left(a_{i}+t\right),(1 \leqq i \leqq 3)$, for some $t$ with $a_{3}+t<n$, then $|\mathscr{A}| \leqq 2^{1-3}$.

Note that the intersection property described in Theorem 5 is similar but not the same as Property $\left(\mathrm{I}_{3}\right)$, since we are not allowing the indice arithmetic to be taken modulo $l$.

\section{Proofs}

Before proving Theorem 1, a special case which will be used in the proof, is to be considered.

Lemma 6. For $l=n \leqq 2 k$, let $X_{1}, X_{2}, \ldots, X_{l}$ be a partition of $X=\{1,2, \ldots, n\}$ into 1 one-element subsets. If $\mathscr{A}$ satisfies $\left(\mathrm{I}_{1}\right)$, then $|\mathscr{A}| \leqq 2^{1-k}$.

Proof. For convenience we will assume $X_{i}=\{i\}$ for each $i,(1 \leqq i \leqq n)$. Also, all indices will be taken modulo $n$. Suppose the lemma is false and that $|\mathscr{A}|>2^{n-k}$. Let $Y$ be the elements of $X$ on which all functions of agree. Thus $|Y|<k$ and $|X-Y|>n-k$, for otherwise $|\mathscr{A}| \leqq 2^{y-k}$.

We claim that if $n-k \leqq|i-j| \leqq k$ for $i, j \in X$ then either $i \in Y$ or $j \in Y$. We assume this is not the case (i.c. $i, j \neq Y$ ) and show that this leads to a contradiction. With no loss of generality we can select $f, g \in \mathscr{A}$ such that $f(i)=0$ and $f(j) \neq g(j)$. Since there are no $k$ integers between $i$ and $j$ ( $j$ and $i$ ), $g(i)=0$. Because $i \notin Y$, there is an $h \in \mathscr{A l}$ such that $h(i)=1$, and we can assume that $h(j) \neq f(j)$. Therefore the pair $f, h$ does not satisfy $\left(\mathrm{I}_{1}\right)$, which completes the proof of the claim.

It follows that for each $i \notin Y$, there are $2 k-n+1$ consecutive integers in $Y$, namely $i+n-k, i+n-k+1, \ldots, i+k$. Therefore a single element in $X-Y$ implies that $2 k-n+1$ elements are in $Y$, and each additional element in $X-Y$ gives an additional element in $Y$. Hence $|Y| \geqq 2 k-n+|X-Y|>2 k-n+n-k \geqq k$, a contradiction, which completes the proof of the lemma.

With the preceding lemma we are now prepared to prove Theorem 1.

Proof (Theorem 1). For $l=l k+r, 0 \leqq r<k$, partition the index set $\{1,2, \ldots, l)\}$ into $k+r$ subsets $\left\{Y_{i j i=1}^{k+p}\right.$ by letting $Y_{i}=\{i, k+i, \ldots,(t-1) k+i\}$ for $1 \leqq i \leqq k$ and $Y_{i+k}=\{i k+i\}$ for $1 \leqq i \leqq r$. Note that any two distinct integers in the same term of this partition differ by at least $k$, so any $k$ consecutive positive intęers modulo $l$ will be in $k$-consecutive terms of 1 his partition modulo $k+r$. Let $W_{1}, W_{2}, \ldots, W_{k+r}$ be the partition of $X$ defined by $W_{i}=\bigcup_{j \in Y_{1}} X_{j}$ for $1 \leqq i \leqq k+r$. Due to the choice of the $Y_{i}^{\prime} s, f, g \in \mathscr{A}$ implies that there is a sequence $s_{b}, s_{b+1}, \ldots$ $\ldots, s_{b+k-1}$ (indices taken modulo $r+k$ ) with $s_{i} \in W_{i}$ such that $f\left(s_{i}\right)=g\left(s_{i}\right)$ for $b \leqq i \leqq b+k-1$.

Partition the $2^{1}$ elements of $\mathscr{F}$ into $2^{1-r-k}$ classes as follows. For fixed $A_{1} \subseteq W_{i}$, $(1 \leqq i \leqq r+k)$ let $S\left(A_{1}, A_{2}, \ldots, A_{r+k}\right)$ be those functions in $\mathscr{F}$ which are constant on $A_{i}$ and $W_{i}-A_{i}$ but have different values on these sets. Clearly $\mid S\left(A_{1}, A_{2}, \ldots\right.$ $\left.\ldots, A_{r+k}\right) \mid=2^{r+k}$ and $\mathscr{F}$ can be partitioned into $2^{1-r-k}$ sets of this type. 
Let $\mathscr{F}^{*}$ be the set of all functions from $\{1,2, \ldots, r+k\}$ into $\{0,1\}$, and let $\tau$ the map from $S=S\left(A_{1}, A_{2}, \ldots, A_{r+k}\right) \cap \mathscr{A}$ into $\mathscr{F}^{*}$ defined by mapping $f$ into $f^{*}$, where $f^{*}(i)=f\left(A_{i}\right)$ for each $i$. Denote the image set of $S$ under the one-to one map $\tau$ by $S^{*}$. Then $S^{*}$ satisfies $\left(\mathrm{I}_{1}\right)$ (where $X=\{1,2, \ldots, k+r\}$ and $S^{*}=\mathscr{A}$ ), so $\left|S^{*}\right| \leqq 2^{r}$ by Lemma 6 . This last inequality is valid for each of the $2^{n-k-r}$ classes of the partition of $\mathscr{A}$. Therefore we have $|\mathscr{A}| \leqq 2^{n-k-r} 2^{r}=2^{n-k}$. Theorem 3.

We need some additional background and a counting result before proving

For $0<t \leqq n-1$ and $r=[t / 2]$, let $B_{1} \subseteq F$ be those functions with at least $n-r$ values equal to 1 and $B_{2} \subseteq F$ those functions with value 0 at a fixed point of the domain and precisely $n-r-1$ values equal to 1 . Set

Then

$$
B=\left\{\begin{array}{lll}
B_{1} & \text { for } t \text { even } \\
B_{1} \cup B_{2} & \text { for } t \text { odd }
\end{array}\right.
$$

$$
|B|= \begin{cases}\sum_{i=0}^{n}\left(\begin{array}{l}
n \\
i
\end{array}\right) & \text { for } t \text { even } \\
\sum_{i=0}^{\infty}\left(\begin{array}{l}
n \\
i
\end{array}\right)+\left(\begin{array}{c}
n-1 \\
r
\end{array}\right) & \text { for } t \text { odd. }\end{cases}
$$

For $f, g \in \mathscr{F}$ we define the distance between $f$ and $g$, denoted $d(f, g)$, to be the number of elements of $X$ on which $f$ and $g$ differ.

Theorem 7 [1]. Let $t$ be fixed, $0<t \leqq n-1$, and let $B$ be defined as above. If $C \leqq \mathscr{F}$ with $d(f, g) \leqq t$ for all $f, g \in C$, then $|C| \leqq|B|$.

Proof (Theorem 3). Assume $\mathscr{A} \subseteq \mathscr{F}$ satisfies property $\left(\mathrm{I}_{2}\right)$. For $1 \leqq i \leqq l$ let $A_{i} \subseteq X_{i}$ and define $S\left(A_{1}, A_{2}, \ldots, A_{t}\right)$ as the set of functions in $\mathscr{F}$ which have a constant value on $A_{i}$ and a different but constant value on $X_{i}-A_{i}$. Clearly $S\left(A_{1}, A_{2}, \ldots, A_{l}\right)$ has cardinality $2^{l}$ and $\mathscr{F}$ can be partitioned into $2^{n-l}$ sets of this type. From the condition $\left(\mathrm{I}_{2}\right)$ satisfied by $\mathscr{A}$, it follows that $S=S\left(A_{1}, A_{2}, \ldots, A_{1}\right) \cap \mathscr{A}$ can be naturally identified with a collection of two valued functions on $l$ points such that the distance between any pair is at most $l-k$. Therefore by Theorem 7 ,

and

$$
|S| \leqq \begin{cases}\sum_{i=0}^{r}\left(\begin{array}{l}
l \\
i
\end{array}\right) & \text { for } l-k=2 r \\
\sum_{i=0}^{r}\left(\begin{array}{l}
l \\
i
\end{array}\right)+\left(\begin{array}{c}
l-1 \\
r
\end{array}\right) & \text { for } l-k=2 r+1,\end{cases}
$$

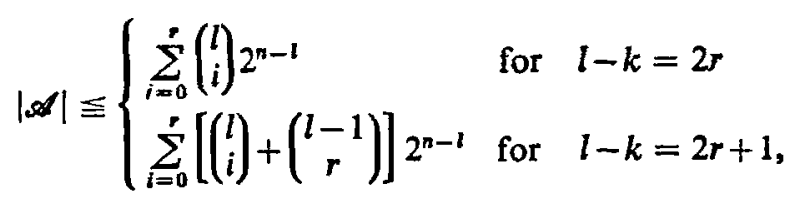

which completes the proof. 
Proof (Theorem 5). Let $a=a_{2}-a_{1}$ and $b=a_{3}-a_{2}$. Consider the graph $G$ with vertex set $X=\{1,2, \ldots, n\}$ and edॄe set $E$ where $x y$ is an ed $\varepsilon$ if and only if $|x-y|=$ $=a, b$ or $a+b$. Using induction on the number of vertices it is easy to verify that $G$ is 4-colorable. Let $X_{1}, X_{2}, X_{3}, X_{4}$ be the four color classes under some 4-coloring of $G$.

By assumption, for each pair $f, g \in \mathscr{A}$ there is an intęer $t$ such that $f$ and $g$ agree on $a_{1}+t, a_{1}+t+a$ and $a_{1}+t+a+b$ respectively, so all of these vertices are pairwise adjacent in the graph $G$. Hence they are elements of different members of the partition $X_{1}, X_{2}, X_{3}, X_{4}$ of $X$. Applying Theorem 3 with $l=4$ and $k=3$ (thus $r=0$ ). we have

$$
|\mathscr{A}| \leqq\left[\left(\begin{array}{l}
4 \\
0
\end{array}\right)+\left(\begin{array}{l}
3 \\
0
\end{array}\right)\right] 2^{n-4}=2^{n-3} \text {. }
$$

The argument used in the above proof can be applied for $k>3$, but unfortunately weaker upper bounds than $2^{n-k}$ are obtained.

\section{Problems}

In addition to the problem raised in the conjecture stated in the introduction, there are several interesting open questions. We will mention two of them.

One of the most obvious questions deals with replacing the family $\mathscr{F}$ of 2 -valued functions by a family of $t$-valued functions for some $t \geqq 3$. For this family of functions, what would be the results analogous to those given Theorems 1 and 3 ?

In both Theorems 1 and 3 examples were given of a subset $\mathscr{A}$ of $\mathscr{F}$ of maximum cardinality satisfying the appropriate intersection property. Is it possible to find all such subsets $\mathscr{A}$ of maximum cardinality? In Eeneral there is not a unique $\mathscr{A}$ of maximum cardinality. For example we can exhibit a family $\mathscr{A} \subseteq \mathscr{F}$ with $|\mathscr{A}|=2^{-k}$ which satisfies $\left(\mathrm{I}_{3}\right)$, but which is distinct from the family described in the introduction. Assume $n=m \cdot l$ where $m \geqq 3, m$ odd. Partition $X$ into $l$ sets $X_{1}, X_{2}, \ldots, X_{1}$, each of cardinality $m$. Let $\mathscr{A}$ be the functions $\mathscr{F}$ with domain $X$ which have at least $(m+1) / 2$ values equal to 1 on each of the sets $X_{i}$ for $1 \leqq i \leqq k$. It is easily verified that $\mathscr{A}$ satisfies $\left(\mathrm{I}_{1}\right)$ and $|\mathscr{A}|=2^{n-k}$.

\section{References}

[1] R. Ahl.swede and G. O. H. Katona, Contributions to the geometry of Hamming spaces, Discrete Math. 17 (1977), 1-22.

[2] N. G. DE BruIJn and P. ERdös, On a combinatorial problem, Proc. Konink Nederland Akad. Wetensch. Amsterdom 51 (1948), 421-423.

[3] F. R. K. Chung, R. L. Graham, P. Frankl and J. Shearer, Some intersection theorems for ordered sets and graphs, to appear.

[4] P. Erdös, ChAo Ko and R. Rado, Intersection theorems for systems of finite sets, Quart. J. Mah. 2(1961), 313-320.

[5] R. L. Graham, M. Simonovits and V. T. Sós, A note on the intersection properties of subsets of integers, J. Comb. Th. (A) 28 (1980), 106-116.

[6] V. RöDL, to appear. 
[7] M. Simonovits and V. T. Sós, Intersections on structures, Combinatorial Math., Optimal Design and their Applications, Ann. Discrete Math. 6 (1980), 301-314.

[8] M. Simonovits and V. T. Sós, Intersection theorems for subsets of integers, European Journal of Comb. 2 (1981), 363-372.

[9] M. Simonovits and V. T. Sós, Graph intersection theorems, Proc. Colloq. Combinatorics and Graph Theory, Orsay, Paris, 1976, 389-391.

[10] M. Simonovirs and V. T. Sós, Intersection theorems for graphs II, Coll. Math. Soc. J. Bolyai 18, Combinatorics, Keszthely, Hungary (1976), 1017-1029.

R. J. Faudree, R. H. Schelp

Department of Mathematical Sciences

Memphis State University

Memphis, Tennessee 38152

USA
V. T. Sós

Mathematical Institute of the

Hungarian Academy of Sciences

Burdapest, Reáltanoda u. 13--15.

H-I364 Hungary 